\title{
Combination of Nitric Oxide Release and Surface Texture for Mitigating Foreign Body Response
}

Authors: Maggie J. Malone-Povolny, Taron M. Bradshaw, Elizabeth P. Merricks, C. Tyler Long, Timothy C. Nichols, and Mark H. Schoenfisch

\section{SUPPORTING INFORMATION}

Synthesis of RSNO-functionalized MSNs. The synthesis of mesoporous silica nanoparticles (MSN) was achieved by combining $175 \mathrm{~mL}$ EtOH, $162 \mathrm{~mL} \mathrm{H}_{2} \mathrm{O}, 11.8 \mathrm{~mL} \mathrm{NH} \mathrm{NH}_{4}$, and $280 \mathrm{mg}$ cetyltrimethylammonium bromide (CTAB).[1,2] This solution was stirred for $\sim 15 \mathrm{~min}$ to form the liquid crystal template necessary for the generation of porous particles. Tetraethylorthosilicate (TEOS, $1.395 \mathrm{~mL}$ ) was added as a bolus with additional stirring $(2 \mathrm{~h})$ at room temperature. The solution became cloudy white, indicating particle formation. Particles were washed three times with ethanol and collected by centrifugation. Following formation of the TEOS MSNs, CTAB still present in the pores was removed via an ion exchange with ethanolic hydrochloric acid. Particles were dried under vacuum, then treated with oxygen plasma for $2 \mathrm{~h}$ to expose surface silanols, facilitating maximum functionalization in subsequent steps.

To functionalize the MSNs with thiol groups, 3-mercaptopropyltrimethexysilane (MPTMS) was surface grafted onto the bare TEOS MSN scaffold. Approximately $50 \mathrm{mg}$ of MSNs were suspended in $20 \mathrm{~mL}$ anhydrous DMF, followed by the addition of $1 \mu \mathrm{L}$ triethylamine/mg MSN. The solution was sonicated to distribute the particles before adding $130 \mu \mathrm{L}$ MPTMS/mg MSN as a bolus. The flask was immediately set to reflux at $150{ }^{\circ} \mathrm{C}$ for $12 \mathrm{~h}$. The solution was then cooled to room temperature, and particles were collected by centrifugation with ethanol wash three times, and dried under vacuum. 
Particles were nitrosated via exposure to acidified nitrite. Thiol-modified particles (25 mg) were dissolved in a mixture of $5 \mathrm{~mL} \mathrm{MeOH}$ and $1 \mathrm{~mL} \mathrm{HCl}(5 \mathrm{M})$. An aqueous solution $(500 \mu \mathrm{L})$ of sodium nitrite $(50 \mathrm{mg})$ and di DTPA $(10 \mathrm{mg})$ was then added slowly to the particle solution. This mixture was shielded from light and stirred at $0{ }^{\circ} \mathrm{C}$ for $1 \mathrm{~h}$. Particles were then collected by centrifugation and washed with $-20^{\circ} \mathrm{C} \mathrm{MeOH}$ three times. The particle batches were dried under vacuum for $45 \mathrm{~min}$ to remove all remaining $\mathrm{MeOH}$. Nitrosated particles, deep pink in color, were used immediately after removal from the vacuum box. Care was exercised to minimize ambient light exposure during the nitrosation process, as light may prompt the premature release of NO.

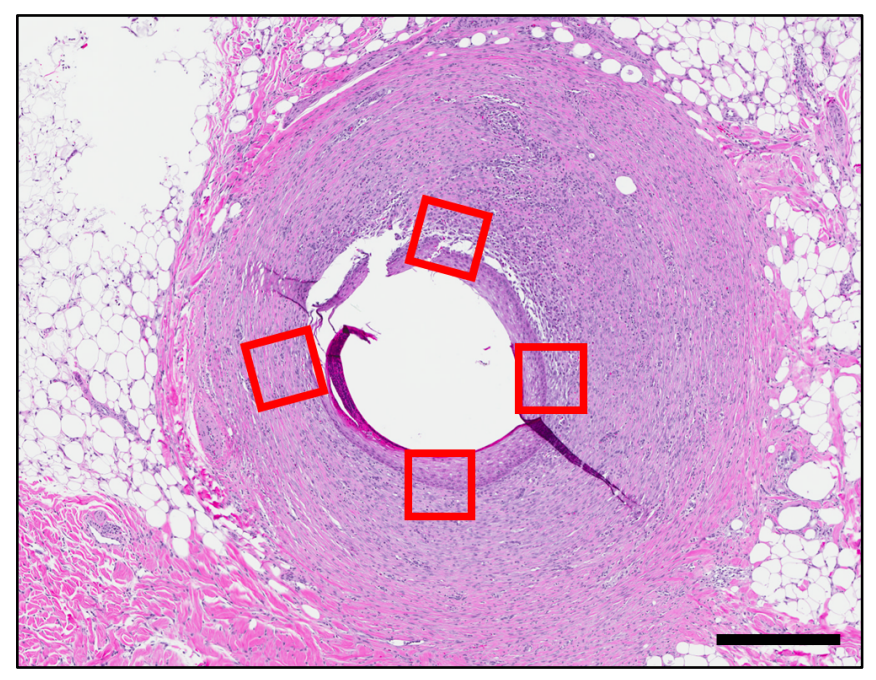

Figure S1: Histological image of hematoxylin and eosin-stained tissue with the areas representative of those selected for tissue analysis highlighted (red boxes). Scale bar represents $400 \mu \mathrm{m}$. 

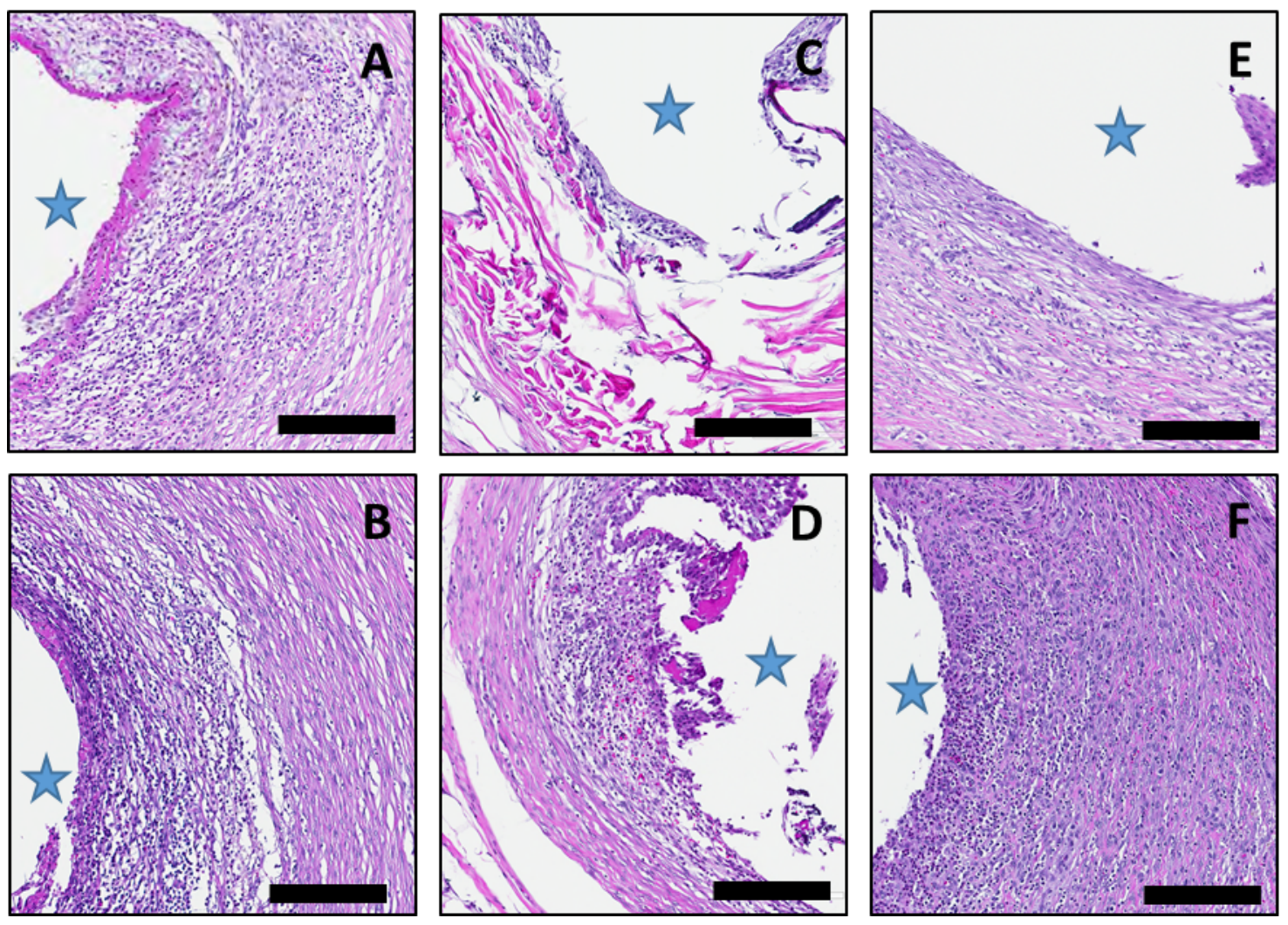

Figure S2: Representative hematoxylin and eosin-stained histological images for (A) smooth NO-releasing; (B) fibercoated NO-releasing; (C) foam-coated NO-releasing; (D) smooth control; (E) fiber-coated control; and (F) foam-coated control implants. Site of implant is marked with a star. Scale bar represents $200 \mu \mathrm{m}$.

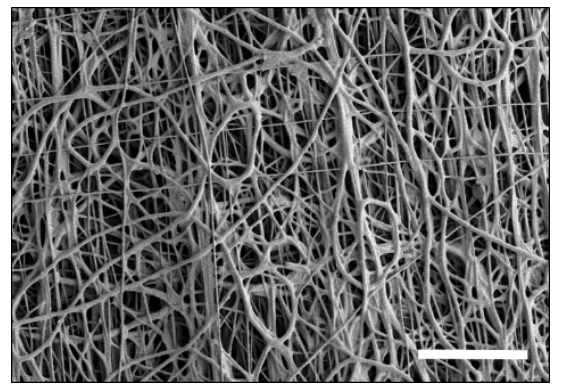

Figure S3: Scanning electron micrograph of surface of fiber-coated polyurethane implant. Scale bar represents 200 $\mu \mathrm{m}$. 


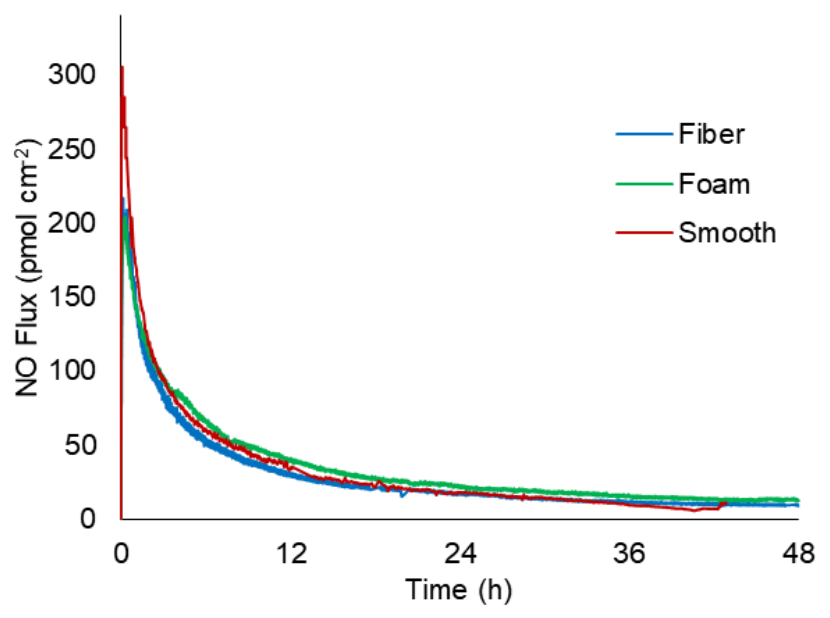

Figure S4: Representative 24-h NO-release profiles of RSNO MSN-doped polyurethane topped with fiber topcoat (blue), foam topcoat (green), or no topcoat (red), as measured by chemiluminescence in $\mathrm{PBS}$ at $\mathrm{pH} 7.4,37^{\circ} \mathrm{C}$, in the dark. 


\section{REFERENCES}

[1] R.J. Soto, L. Yang, M.H. Schoenfisch, Functionalized Mesoporous Silica via an Aminosilane Surfactant lon Exchange Reaction: Controlled Scaffold Design and Nitric Oxide Release, ACS Appl. Mater. Interfaces. 8 (2016) 2220-2231. https://doi.org/10.1021/acsami.5b10942.

[2] M.J. Malone-Povolny, M.H. Schoenfisch, Extended Nitric Oxide-Releasing Polyurethanes via S-Nitrosothiol-Modified Mesoporous Silica Nanoparticles, ACS Appl. Mater. Interfaces. 11 (2019) 12216-12223. https://doi.org/10.1021/acsami.8b19236. 